\title{
Universités : innover ou sombrer, par P.
} Fauconnier

\section{Charlotte Pourcelot}

\section{(2) OpenEdition}

1 Journals

Édition électronique

URL : http://journals.openedition.org/ripes/1929

DOI : 10.4000/ripes.1929

ISSN : 2076-8427

Éditeur

Association internationale de pédagogie universitaire

Référence électronique

Charlotte Pourcelot, « Universités : innover ou sombrer, par P. Fauconnier », Revue internationale de pédagogie de l'enseignement supérieur [En ligne], 35(1) | 2019, mis en ligne le 19 mai 2019, consulté le 25 septembre 2020. URL : http://journals.openedition.org/ripes/1929 ; DOI : https://doi.org/10.4000/ ripes. 1929

Ce document a été généré automatiquement le 25 septembre 2020.

Article L.111-1 du Code de la propriété intellectuelle. 


\section{Universités : innover ou sombrer, par P. Fauconnier}

\section{Charlotte Pourcelot}

1 En France, l'échec au premier cycle universitaire, la difficile insertion professionnelle des jeunes diplômés, la baisse du taux de rendement des diplômes et le phénomène d'inflation scolaire ont tendance à dévaloriser les formations universitaires généralistes au profit des formations professionnalisantes et des filières prestigieuses des grandes écoles. L'enseignement supérieur français semble donc tourmenté, raison pour laquelle le gouvernement, élu en mai 2017, entend accompagner chaque apprenant vers la réussite grâce à une nouvelle réforme qui met en œuvre le «Plan Etudiants » et la loi « Orientation et réussite étudiantes ».

2 Dans ce contexte, le dernier ouvrage de Patrick Fauconnier (2017), journaliste français spécialiste de l'éducation et plus précisément de l'enseignement supérieur, interpelle de par la marche à suivre qu'il indique : "Universités : innover ou sombrer ». Ce manuscrit, bien que non-scientifique et non-académique, mérite toutefois que l'on y porte attention bien qu'il ne soit pas destiné aux spécialistes ${ }^{1}$. En tant que chercheure en sciences de l'éducation, il nous paraît pertinent de nous y intéresser et d'une certaine façon, d'y répondre au travers de cette note de lecture, car des sujets multiparamétriques et complexes à appréhender y sont abordés.

3 L'ouvrage se compose d'une partie introductive, de trois volets et d'une partie conclusive. Il ambitionne d'analyser la dualité du système d'enseignement supérieur, avec d'un côté, les grandes écoles et de l'autre, l'université. Au-delà du constat de nonefficience, l'auteur propose quatre mesures à mettre en œuvre dans un court délai pour rentrer dans la course des classements internationaux et bonifier l'université française pour que celle-ci puisse renaître et rayonner, deux objectifs urgents selon lui. Le manuscrit est foisonnant - nous noterons au passage l'absence de bibliographie et de sitographie $^{2}$ - le but de cette contribution n'est donc pas de rapporter de manière exhaustive les propos et les idées qui y sont exposées mais d'en pointer d'abord les éléments saillants pour en proposer ensuite une analyse et ouvrir le débat. 
4 L'introduction est principalement ancrée dans les champs économique, politique et historique. Tous trois constituent d'ailleurs la toile de fond de l'ouvrage. Elle entend retracer les difficultés rencontrées ces huit dernières décennies par la population française à savoir : un "taux de chômage record", une "industrie dévastée ", un "commerce extérieur en berne », une "dette abyssale», « 8 millions de pauvre» (Fauconnier, 2017, p. 14), et ce, sans langue de bois comme en témoignent ces quelques extraits. Selon le journaliste, les établissements d'enseignement sont la cause de notre «panade actuelle » (Fauconnier, 2017, p. 15). Il rappelle qu'« en Allemagne, en Suisse et en Autriche, l'enseignement supérieur est un puissant vecteur de formation professionnelle, par le biais d'universités techniques et de «polytechnicum» mondialement renommés, facteur de plein emploi dans ces pays " (Fauconnier, 2017, p. 16). Dès les premières pages, le lecteur saisit que le scindement universités/grandes écoles ne rendrait pas service à ses usagers et la première partie de l'ouvrage y est consacrée.

5 La première partie intitulé « Un enseignement supérieur inefficient » vise à dépeindre l'ensemble des faiblesses de l'université. Avec certaines longueurs parfois, Fauconnier (2017) décrit l'état de délabrement que connaissent certains campus notamment à cause d'un manque d'argent et de cohérence architecturale. Les campus universitaires français, " constitués d'alignements massifs de blocs de béton organisés à angles droits comme des casernes» (Fauconnier, 2017, p. 30) et «mornes» (Fauconnier, 2017, p. 31) ne sont pas louables. Avec raison, l'auteur plaide pour «de vrais campus interdisciplinaires » (Fauconnier, 2017, p. 33), sans division, auxquels seraient rattachés les Instituts Universitaires de Technologie fréquemment géographiquement éloignés. Selon lui, les campus ont besoin d'être repensés pour ne plus être éparpillés et pouvoir générer un sentiment d'appartenance (Mucchielli, 1980) nécessaires à la réussite des usagers. Nous notons au passage que le journaliste ne fait pas allusion à la présence de cellule dédiée à la vie du campus et à la vie étudiante dans la plupart des établissements, preuve selon nous que les gouvernances ont compris l'enjeu et l'importance de ces facteurs et qu'elles s'en préoccupent avec les moyens dont elles disposent.

6 Fauconnier (2017) énonce ensuite ce qui selon lui a été préjudiciable pour l'enseignement supérieur français. Nous retiendrons notamment: l'éclatement de certaines universités renommées comme celle de La Sorbonne, « l'irruption de stupides appellations technocratiques "Paris-1, Paris-2, etc." » (Fauconnier, 2017, p. 37), la nomination de nombreux hommes et femmes politiques au ministère de l'Enseignement Supérieur et de la Recherche ${ }^{3}$, et la succession de politiques éducatives. "Ces "ratés" à répétition sont autant de preuves éclatantes que nos hauts dirigeants, issus pour la majorité des grandes écoles et des grands corps, se sont moqués de l'université » (Fauconnier, 2017, p. 59). En somme, la première partie de l'ouvrage s'attache à démontrer que "l'université et la science reste les parents pauvres du système » (Fauconnier, 2017, p. 76) et à illustrer le «mépris des gouvernants à l'égard des universités » (Fauconnier, 2017, p. 77).

7 S'agissant de l'enseignement, l'auteur note également l'absence d'intérêts pour des champs pourtant porteurs d'emplois comme le design, la mode, le luxe, la criminologie ou encore le renseignement. Il est toutefois regrettable que la place de l'enseignement, l'une des trois fonctions principales de l'enseignant-chercheur avec la recherche et l'administration - rappelons-le - ne soit éluder qu'en quelques pages, et qu'aucune 
solution ne soit proposée pour valoriser l'acte d'enseigner et par la même occasion, la qualité de l'enseignement universitaire. En effet, les différents facteurs qui pourraient expliquer le désintérêt de certains pour l'enseignement ne sont pas présentés, Fauconnier (2017) rappellant uniquement que les enseignants-chercheurs sont "soumis à la dictature du "publish or perish" " (Fauconnier, 2017, p. 82) sans contextualiser et sans émettre de pistes pour que l'enseignement soit (re)considéré. Nous ne pouvons que regretter la réflexion s'arrête à ce constat.

Concernant les grandes écoles, les comparaisons avec le système anglo-saxon sont récurrentes. Selon le journaliste, pour plus de cohérence et d'efficience, il conviendrait de rapprocher les grandes écoles et de rompre la culture communautaire. Il plaide ainsi pour une ouverture à un plus grand nombre d'étudiants d'origines diverses, la diversification des modes de recrutement et le développement de cours relatifs au «behavior».

9 La deuxième partie au titre prescriptif «[i]l faut réunifier notre enseignement supérieur» dénonce le morcellement et la fragmentation des établissements d'enseignement et de recherche, et invite à une réflexion sur à la mutualisation des atouts de ces sites. Selon l'auteur, une fusion permettrait de former un "Cambridge français» (Fauconnier, 2017, p. 150) qui pourrait être un important moteur de puissance économique et de rayonnement scientifique. Fauconnier (2017) milite pour le rapprochement des universités, des grandes écoles et des entreprises, et l'émergence d'une culture commune à ces trois entités. L'université est aussi invitée à s'impliquer dans la formation professionnelle. L'auteur évoque ainsi les Initiatives D'EXcellence (IDEX) dont le but est de créer sur le territoire français des ensembles pluridisciplinaires d'enseignement supérieur et de recherche de rang mondial. Il cite également les Initiatives Science Innovation Territoires Économie (ISITE) initiées par la loi n²013-660 du 22 juillet 2013 relative à l'enseignement supérieur et à la recherche qui promeut l'émergence de pôles universitaires et de recherche de taille nationale et internationale grâce aux actions volontaristes des Programmes d'Investissements d'Avenir (PIA). Selon le journaliste, "notre pays a tout à gagner à ce qu'écoles, universités et organismes de recherche progressent en réputation en unissant leurs moyens, afin d'obtenir un meilleur soutien des milieux économiques» (Fauconnier, 2017, p. 191-192).

10 Le vocabulaire parfois fleuri en dit long sur l'exaspération de l'auteur et son intention de dénoncer un système déficient. Militant, en sa qualité d'analyste et d'expert, il plaide en faveur d'un pôle scientifique d'envergure mondiale et pour la constitution d'universités capables de se hisser au rang des meilleures. Il rappelle d'ailleurs à plusieurs reprises l'absence d'établissements français dans les classements internationaux (Palmarès de Shanghai et Palmarès du Times ${ }^{4}$ ). L'approche comparatiste à l'échelle européenne et mondiale invite les acteurs de l'enseignement supérieur à s'allier pour créer une stratégie enseignement/recherche qui reposerait sur le renforcement de son attractivité grâce à l'internationalisation de l'université et sa professionnalisation. "C'est un fait: notre enseignement supérieur affiche un ahurissant éparpillement de ses structures de formation, causant une fragmentation des ressources qui entrave tout le rendement du système " (Fauconnier, 2017, p. 204). Ces propos peuvent égarer un lecteur peu sensible à ces critères, fussent-ils accompagnés d'une analyse économique comme ici. 
11 La troisième partie intitulée «Les quatre mesures à prendre vite » prescrit: une propédeutique et des "colleges " comme aux USA; une université évaluée et notée, payante et démocratique, et enfin sélective. Comment? L'auteur suggère d'harmoniser les pratiques académiques des universités, des grandes écoles et des grands organismes en mettant fin à la fracture culturelle qui les caractérise. L'auteur suggère une refondation du système d'enseignement supérieur qui doit commencer dès le lycée et concerner la formation et l'orientation des néo-bacheliers. La première année universitaire devrait ainsi devenir une année propédeutique interdisciplinaire et sans sélection, consacrée à l'apprentissage des méthodes de travail propres à l'enseignement supérieur et à l'élaboration d'une orientation. Un nouveau corps d'enseignants, exclusivement dévolu au premier cycle, devrait alors être envisagé. Ces solutions peuvent s'entendre, néanmoins s'agissant de la notation des universités par les étudiants pour aider à l'orientation, nous pensons qu'il s'agit d'un leurre et qu'elle ne conduirait qu'à la mise en concurrence des établissements dont les plus petits souffriraient. Dans cette partie, nous aurions grandement apprécié que l'humain soit au cœur de l'argumentaire et que des outils comme l'accompagnement ou la coopération fassent partie des mesures à adopter en urgence.

12 Fauconnier (2017) adopte un discours franc et surtout exempt de toute rhétorique. Sa force argumentaire réside en partie dans les anecdotes et le ton sarcastique pour lequel il opte régulièrement et qui participe à révéler toute la complexité et les faiblesses de l'enseignement supérieur avec originalité. Avec ses lunettes d'économiste, il dénonce avec conviction les aberrations du système d'enseignement supérieur français, propose, va droit au but et soutient à sa manière la fierté universitaire en délivrant un message d'espoir dont on peut toutefois s'étonner et regretter que le vocable " enseignement » soit quasiment absent. L'auteur accuse, récuse. Cet ouvrage secoue, bouscule, parce qu'il recense de nombreux problèmes que cumulent l'enseignement supérieur français. Fauconnier (2017) se fait le porte-voix de ses handicaps et suggère un autre mode de fonctionnement, fondé sur le rapprochement des grandes écoles et des universités pour améliorer son rayonnement scientifique et technologique, clé de la performance académique selon lui.

13 Toutefois, certains propos en faveur d'un système réunifié ne sont pas suffisamment nuancés, plusieurs informations affirmatives ne comportent aucune source, ce qui laisse planer le doute. Et les propos, parfois très orientés, laissent entendre une transformation du système éducatif en un business à inscrire dans la «compétition internationale » (Fauconnier, 2017, p. 232), avis que nous ne partageons pas. L'ouvrage est aussi parfois répétitif. À plusieurs reprises le lecteur a l'impression de «tourner en rond» et de ne pas progresser dans l'argumentaire, d'autant que les lacunes sont immédiatement adossées à des actions solutives pas toujours concrètes et difficilement applicables.

14 En tant que chercheure en sciences de l'éducation, la principale limite de l'ouvrage et non des moindres, est l'absence de recours aux travaux scientifiques. Hormis François Dubet (Fauconnier, 2017, p. 184), Christine Musselin (Fauconnier, 2017, p. 199) et François Garçon (Fauconnier, 2017, p. 220), les éminents travaux menés par des chercheurs de renommée internationale comme: Emmanuelle Annoot, Denis Berthiaume, Laurent Cosnefroy, Amaury Daele, Marie Duru-Bellat, Marianne Frenay, Geneviève Lameul, Joëlle Demougeot-Lebel, Christelle Lison, Christophe Michaut, Sophie Morlaix, Saeed Païvandi, Cathy Perret, Nicole Poteaux, Marianne Poumay, 
Sophie Orange, Marc Romainville, Agnès Van Zanten, Joël Zaffran - et bien d'autres encore - sont passés sous silence alors qu'ils mériteraient d'être investis dans un tel ouvrage, aussi vulgarisé soit-il. L'auteur occulte d'importantes contributions fondées sur la recherche qui questionnent, en autres, les politiques éducatives propres à l'enseignement supérieur et qui contribuent à son amélioration. La place manque ici pour développer les apports des sciences de l'éducation dans ces domaines complexes, mais ceux-ci existent et méritent d'être portés à la connaissance des "non-initiés ».

Pour clore cette note de lecture, nous retiendrons principalement la nécessité de faire renaître une fierté universitaire en France, message résumé par André Brahic qui soutient qu'il y a trois priorités absolues pour le pays : l'éducation, la recherche et la culture.

\section{BIBLIOGRAPHIE}

Fauconnier, P. (2017). Universités : innover ou sombrer. Paris, France : Fauves.

Mucchielli, R. (1980). Le travail en groupe. Paris, France : Éditions ESF.

\section{NOTES}

1. En septième page, l'auteur avertit le lecteur que l'ouvrage ne s'adresse pas aux spécialistes mais aux non-initiés.

2. Il est aussi regrettable de relever plusieurs fautes d'orthographe/de frappe et l'absence de signes de ponctuation.

3. Notons que depuis l'élection présidentielle de 2017, ce ministère s'appelle "Ministère de l'Enseignement Supérieur, de la Recherche et de l'Innovation ».

4. « Aujourd'hui encore, la sélection reste, avec la scolarité payante, et le système de nomination des profs, l'un des trois derniers verrous qui barrent l'accès de l'université française aux standards internationaux » (p. 66).

\section{AUTEUR}

\section{CHARLOTTE POURCELOT}

Université Paris-Est, Marne-la-Vallée, France.

charlotte.pourcelot@univ-paris-est.fr 